\title{
Tupaiine tree shrews (Scandentia, Mammalia) from the Yuanmou Lufengpithecus locality of Yunnan, China
}

\author{
Xijun Ni · Zhuding Qiu
}

Received: 26 September 2011/ Accepted: 27 October 2011/Published online: 11 November 2011

(C) Akademie der Naturwissenschaften Schweiz (SCNAT) 2011

\begin{abstract}
Tree shrew fossils are extremely rare. Here, we report two new tupaiine tree shrews, Prodendrogale engesseri sp. nov. and Tupaia storchi sp. nov., discovered from the late Miocene deposits of Yuanmou Lufengpithecus locality of Yunnan Province in China. P. engesseri is very close to the slightly younger species $P$. yunnanica Qiu (Vertebrata PalAsiatica, 24: 308-319, 1986) from the Lufeng Lufengpithecus locality of Yunnan Province. Relatively lower crowns and less trenchant tooth cusps of $P$. engesseri show that $P$. engesseri is more primitive than $P$. yunnanica. Tupaia storchi is a species larger than Palaeotupaia sivalicus Chopra and Vasishat, 1979, T. minor Günther, 1876 and T. javanica Horsfield, 1822, but smaller than all the other extant species of Tupaia and T. miocenica Mein and Ginsburg, 1997. The mesiobuccal side of the lower molar of this species develops a very strong cingulid. It should be interpreted as a primitive condition. Discovery of diverse tree shrew fossils in Yunnan suggests that multiple evolutionary lineages of tree shrews must have coexisted in a very large area in East Asia.
\end{abstract}

Keywords Prodendrogale $\cdot$ Tupaia $\cdot$ Scandentia . Late Miocene Baodean

\section{Introduction}

Tree shrews are squirrel-like small-bodied mammals living in the South and Southeast Asia. In the 1920s, it was suggested that tree shrews have some characters closely linking them to primates (Carlsson 1922; Le Gros Clark

\section{Ni (凹) - Z. Qiu}

Institute of Vertebrate Paleontology and Paleoanthropology,

Chinese Academy of Sciences, 142 Xi Zhi Men Wai Street,

Beijing 100044, China

e-mail: nixijun@ivpp.ac.cn 1924a, b, 1925, 1926). Later on, the tree shrews were formally transferred from the Order Insectivora to the Order Primates (Simpson 1945). Since 1960s, however, more and more researchers began to challenge the primate status of tree shrews (Martin 1968; McKenna 1966; Szalay 1968; Van Valen 1965). In modern classification, tree shrews are put in an order of their own (Helgen 2005; McKenna and Bell 1997), but most researchers still believe that tree shrews have a close phylogenetic relationship with primates, and great efforts have been poured into the studies on the superordinal phylogenetic relationships among tree shrews, primates, plesiadapiforms and flying lemurs (Bloch et al. 2007; Janecka et al. 2007; Ni et al. 2010; Sargis 2004; Schmitz et al. 2000; Simmons 1993; Springer et al. 2007). There are also many papers dealing with the intra-ordinal relationships of extant tree shrews from multiple aspects (Luckett and Jacobs 1980; Olson et al. 2004, 2005; Steele 1973).

Our understanding on the origin and early evolution of this group is still obstructed by its poor fossil record. Although many fossil mammals from Eurasia and North America, such as Tupaiodon, Adapisoriculus, Litolestes, and Anagale, were suggested to be tree shrews or close relative of tree shrews (Matthew and Granger 1924; Simpson 1931; Szalay 1977; Van Valen 1965), undoubted tree shrew fossils are extremely rare. They were only found in the Siwaliks of India and Pakistan, Li Basin of Thailand, Lufeng and Yuanmou in Yunnan Province, and possibly Xichuan in Henan Province of China.

Some isolated teeth and one jaw fragment collected from the Yuanmou Lufengpithecus locality were attributed to Prodendrogale yunnanica and Ptilocercinae gen. and sp. indet. in a study on the small mammalian fauna of the locality (Ni and Qiu 2002). Detailed examination of the specimens reveals that there are probably four types of tree 
shrews in the Yuanmou Lufengpithecus mammalian fauna. The lower jaw fragment preserving an $\mathrm{m} 1$ and half $\mathrm{p} 4$ was identified as a ptilocercine tree shrew (Ni and Qiu 2002). Restudy of the specimen confirms our previous identification and suggests that it must belong to a new genus and species. One previously unknown lower molar fragment preserving half of the trigonid and complete talonid shows very bunodont cusp pattern and possess weak cingulids on it buccal and distal sides. The tooth is much smaller than the specimen previously referred to Ptilocercinae, but larger and much more bunodont than the specimens allocated to Prodendrogale. The combination of bunodont lower molar with buccal and distal cingulid clearly suggests a ptilocercinae affinity. Detailed study on these ptilocercine specimens is still in process. Here, we focus on the tupaiine specimens only. The isolated teeth previously attributed to $P$. yunnanica are now identified as a new species. Some newly collected specimens from the same locality indicate that a previously unknown Tupaia type tree shrew is also present in the Yuanmou Lufengpithecus mammalian fauna.

\section{Material and method}

The Yuanmou Lufengpithecus locality lies in the Yuanmou Basin, which is one of the numerous Cenozoic sedimentary basins on the Yunnan-Guizhou Plateau in southwest China. It is located near the Leilao, Xiaohe and Zhupeng villages of the Yuanmou County, about $30 \mathrm{~km}$ northwest to the Yuanmou city and $220 \mathrm{~km}$ northwest to the Kunming, capital of the Yunnan Province. The fossil-bearing sediments are mainly diluvial-alluvial deposits, comprising a series of brownish-red sandy clays and argillaceous siltstones, with some intercalation or small lenticular bodies of grayish-yellow and grayish-green pebbly sandstone ( $\mathrm{Ni}$ and Qiu 2002; Qi and Dong 2006).

Specimens described here were obtained by screen washing. More than 10 tons of matrix collected from the Lufengpithecus locality near Leilao Village of Yuanmou County were processed. About two hundred isolated teeth of small mammals representing 41 taxa were sorted out from the concentrates. But the tree shrews are very rare. Only about a dozen specimens were found. Terminology for dental description follows that of Szalay and Delson (1979). Measurements were taken under Olympus SZ61 microscope with a precision of $0.01 \mathrm{~mm}$. Odontometric landmarks follow the definition of Swindler (1976).

\section{Systematic palaeontology}

Class Mammalia Linneaus, 1758

Order Scandentia Wagner, 1855
Family Tupaiidae Gray, 1825

Subfamily Tupaiinae Gray, 1825

Genus Prodendrogale Qiu (1986)

Prodendrogale engesseri sp. nov

Figure 1a-n; Table 1

Holotype Right M1, IVPP V 18215 (2.10 mm long, and $2.35 \mathrm{~mm}$ wide, Fig. 1a).

Type locality Yuanmou Lufengpithecus locality 9906, Xiaohe Formation, Leilao Village, Yuanmou County, Yunnan Province, China. Late Miocene, Early Baodean Chinese Land Mammalian Age, MN10 of Europe.

Etymology Dedicated to Dr. Burkart Engesser, in recognition of his outstanding work on fossil mammals and his important contributions to Chinese mammal palaeontology.

Referred specimens Specimens from Yuanmou Lufengpithecus locality 9906: right P4, IVPP V 18216.1 (1.80 mm long, and $1.62 \mathrm{~mm}$ wide); left P4, IVPP V 18216.2 (1.79 mm long, and $1.65 \mathrm{~mm}$ wide); left M1 fragment, IVPP V 18216.3; right M2 fragment, IVPP V $18216.4(\sim 2.00 \mathrm{~mm}$ long, and $2.35 \mathrm{~mm}$ wide); right M2 fragment, IVPP V 18216.5 ( $\sim 2.40 \mathrm{~mm}$ wide); right M2 fragment, IVPP V 18216.6; left M3, IVPP V 18216.7 (1.65 mm long, and $2.25 \mathrm{~mm}$ wide); right p3 fragment, IVPP V 18216.8 ( $\sim 1.40 \mathrm{~mm}$ long, and $0.55 \mathrm{~mm}$ wide); right $\mathrm{p} 4$, IVPP $\mathrm{V}$ 18216.9 (1.65 mm long, and $0.91 \mathrm{~mm}$ wide); right $\mathrm{p} 4$ fragment, IVPP V 18216.10 (1.72 mm long, and $1.15 \mathrm{~mm}$ wide); right $\mathrm{m} 2$ fragment, IVPP V 18216.11 (2.26 mm long, $\sim 1.40 \mathrm{~mm}$ trigonid wide, and $1.35 \mathrm{~mm}$ talonid wide); left $\mathrm{m} 2$ fragment, IVPP V $18216.12(\sim 2.20 \mathrm{~mm}$ long, and $1.40 \mathrm{~mm}$ trigonid wide); left $\mathrm{m} 3$ fragment, IVPP V 18216.13 ( $\sim 1.80 \mathrm{~mm}$ long, and $1.05 \mathrm{~mm}$ trigonid wide). Specimens from Yuanmou Lufengpithecus locality 9905: left M1, IVPP $\mathrm{V} 18217.1$ (2.20 $\mathrm{mm}$ long, and $2.71 \mathrm{~mm}$ wide); right $\mathrm{M} 1$ or M2 fragment, IVPP V 18217.2; left p4, IVPP V 18217.3 (1.64 mm long, and $1.00 \mathrm{~mm}$ wide); right $\mathrm{m} 2$ fragment, IVPP V 18217.4 (2.20 mm long, $1.45 \mathrm{~mm}$ trigonid wide, and $1.49 \mathrm{~mm}$ talonid wide); right $\mathrm{m} 1$ or $\mathrm{m} 2$ talonid, IVPP $\mathrm{V}$ 18217.5 ( $1.48 \mathrm{~mm}$ talonid wide).

Diagnosis Tree shrew similar to $P$. yunnanica, but with smaller size and lower crown; P4 buccal side possessing deep indentation; parastyle of P4 large; paraconule and metaconule of upper molar small, parastyle and mesostyle moderately protruding; hypoconulid of lower molar large, valley separating entoconid and hypoconulid wide.

Description The occlusal view of the P4 is roughly triangle, but the mesial and buccal margins of the tooth have deep indentation, and its distal edge is waisted. The paracone is very tall and sharp, pronouncedly inclined to the distobuccal side. The mesial margin of the paracone is rounded. The preparacrista extends from the parastyle to the mesial margin of the paracone for a very short distance. The postparacrista bearing on the distal margin of the 


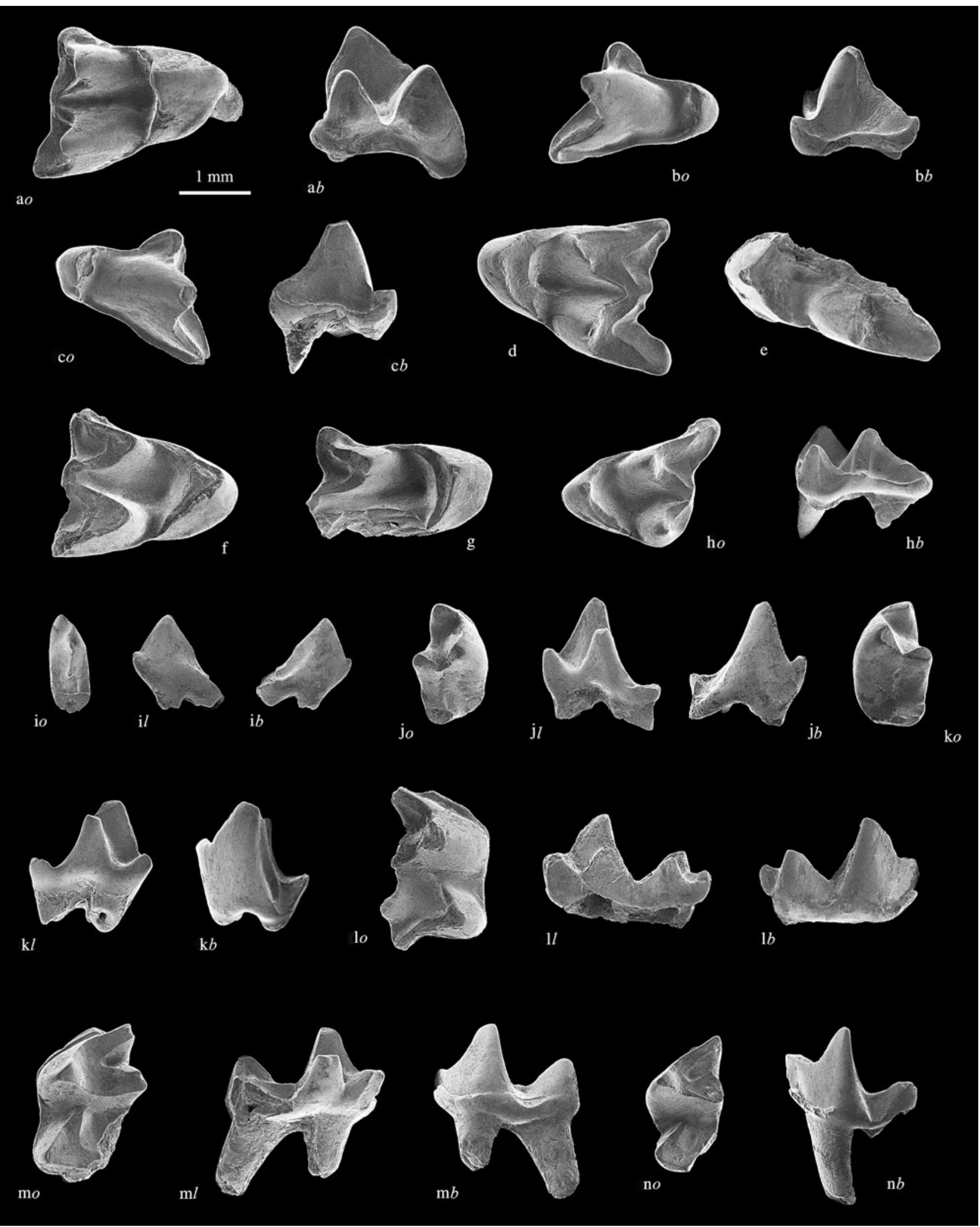

Fig. 1 Isolated teeth of Prodendrogale engesseri sp. nov.: a right M1, IVPP V 18215, type; b right P4, IVPP V 18216.1; c left P4, IVPP V 18216.2; d left M1, IVPP V 18217.1; e left M1 fragment, IVPP V 18216.3; f right M2 fragment, IVPP V 18216.4; $g$ right M2 fragment, IVPP V 18216.5; h left M3, IVPP V 18216.7; i right p3 fragment,
IVPP V 18216.8; j right p4, IVPP V 18216.9; k left p4, IVPP V 18217.3; I right $\mathrm{m} 2$ fragment, IVPP V 18216.11; $\mathbf{m}$ left $\mathrm{m} 2$ fragment, IVPP V 18216.12; n left m3 fragment, IVPP V 18216.13. $o$ occlusal view, $l$ lingual view, $b$ buccal view 
Table 1 Measurements of the isolated teeth of Prodendrogale engesseri sp. nov. and P. yunnanica (in mm)

\begin{tabular}{|c|c|c|c|c|c|c|c|c|}
\hline \multirow[t]{2}{*}{ Specimen } & \multicolumn{4}{|c|}{ Prodendrogale yunnanica } & \multicolumn{4}{|c|}{ Prodendrogale engesseri } \\
\hline & $N$ & Length & Trigon/trigonid width & Talonid width & $N$ & Length & Trigon/trigonid width & Talonid width \\
\hline $\mathrm{C} 1$ & 1 & 1.25 & 0.60 & - & 0 & - & - & - \\
\hline $\mathrm{P} 2$ & 1 & 1.25 & 0.58 & - & 0 & - & - & - \\
\hline P3 & 2 & 1.68 & - & - & 0 & - & - & - \\
\hline P4 & 1 & 1.75 & - & - & 2 & 1.80 & 1.64 & - \\
\hline M1 & 1 & 2.50 & - & - & 2 & 2.15 & 2.53 & - \\
\hline M2 & 1 & 2.25 & 2.60 & - & 2 & 2.00 & 2.38 & - \\
\hline M3 & 1 & 1.50 & 2.60 & - & 1 & 1.65 & 2.25 & - \\
\hline p3 & 0 & - & - & - & 1 & 1.40 & 0.55 & - \\
\hline $\mathrm{p} 4$ & 1 & 1.90 & 1.25 & - & 3 & 1.67 & 1.02 & - \\
\hline $\mathrm{m} 1$ & 2 & 2.45 & 1.40 & 1.60 & 0 & - & - & - \\
\hline $\mathrm{m} 2$ & 1 & 2.40 & 1.40 & 1.35 & 3 & 2.22 & 1.42 & 1.42 \\
\hline $\mathrm{m} 3$ & 1 & - & - & 1.15 & 1 & 1.80 & 1.05 & - \\
\hline
\end{tabular}

paracone is sharp and long. It originates from the tip of the paracone, and extends distolingually to the midway, then turns to the distobuccal corner of the tooth, forming a long and curved shearing blade. The protocone is mesiolingually positioned relative to the paracone, and much lower and narrower than the latter cusp. The lingual side of the protocone is round and bulging, but the buccal side is very steep and slightly concave. The preprotocrista is short and sharp. It starts at the tip of the protocone, and runs mesiobuccally for a very short distance to join the mesial cingulum. The postprotocrista is absent, but the "Nannopithex"-fold (= postprotocone-fold) is about equally developed as the preprotocrista. It is short and confluent with the distal cingulum. The parastyle is very large. It is located mesiobuccally relative to the paracone. Because of the mesial protruding of protocone and parastyle, the indentation of the mesial border of the tooth becomes very prominent. The metastyle is very small, present as a small nodule at the distal end of the postparacrista. Except for the lingual side of the protocone, the whole tooth is surrounded by a very strong cingula. The mesial cingulum and the parastyle enclose a small fossa mesial to the paracone. The buccal cingulum and the distal part of the postparacrista enclose a prominent stylar shelf.

The M1 is also roughly triangular in the occlusal view. The protocone is large and robust. The tip of the protocone is more mesially positioned. The mesiolingual and the distolingual surfaces of the protocone meet lingually at a sharp angle and form a prominent arris. The lingual side of the protocone is deeply concave and bordered by sharp preprotocrista and postprotocrista. The preprotocrista extends to the base of the tooth mesial to the paracone and joins the mesial cingulum. The postprotocrista is slightly longer than the preprotocrista due to the mesial inclination of the protocone. It extends to the tooth base near the distolingual margin of the metacone. A very weak paraconule and metaconule are developed on the preprotocrista and postprotocrista, respectively. The paracone is a triangular-pyramid-like cusp. Its mesiolingual and distolingual surfaces are flat and meet lingually at a sharp angle. A sharp edge is therefore formed on the lingual surface of the cusp. The tip of the paracone inclines buccally. The buccal side of the paracone is deeply concave, with the sharp preparacrista and postparacrista enclosing the fossa. The metacone is in a similar shape as the paracone, but significantly larger. The cristae bearing on the paracone and metacone are arranged in a typically dilambdodont pattern. The preparacrista extends mesiobuccally to join the parastyle. The postparacrista has about the same length as the preparacrista, and extends distobuccally to join the mesostyle. The premetacrista extends mesiobuccally, also joining the mesostyle. The postmetastyle extends distobuccally for a much longer distance. Along the buccal side of the tooth, the stylar shelf is wide. Two stylar cusps are developed, the parastyle and the mesostyle. The parastyle is relatively small and blunt. The mesostyle does not protrude buccally. The weak mesial cingulum connects the preprotocrista and parastyle. On the mesial side of the protocone, a trace of cingulum is developed. But this weak cingulum does not connect the preprotocrista or the mesial cingulum. The distal cingulum is even weaker. It extends along the distal base of the metacone like a trace. On the distolingual side of the protocone, a short but prominent cingulum is developed. Similar as the cingulum on the mesial side of the protocone, this short cingulum developed on the distolingual margin of the protocone does not connect the postprotocrista or distal cingulum.

Complete M2 has not been found. A few fragments show that the tooth is similar to the M1, but more symmetrical from occlusal view. Its postmetacrista is not as 
elongated as the one of M1. The tip of the protocone is more centrally positioned. The parastyle and mesostyle are relatively larger and more protruding. The short cingula on the mesial and distolingual margins of the protocone are absent or very weak.

The M3 is much smaller than M1 and M2. From the occlusal view, it is very asymmetrical: the paracone is fully developed, whereas the metacone is greatly reduced. The mesiobuccal corner of the tooth is elongated into an armlike structure. The preparacrista is also elongated, much longer than the postparacrista. The premetacrista is normally developed, but the postmetacrista is absent. The distal surface of the metacone is thereby rounded and smooth. The protocone is relatively small and elegant. The paraconule and metaconule are absent. The parastyle is pretty large, but the mesostyle is small.

Only one incomplete $\mathrm{p} 3$ is available for description. The tooth is buccolingually compressed. The protoconid is very tall and triangular in the side view. Both the mesial and distal cristids of the protoconid are very sharp. The paraconid is very small, developed at the mesial end of the tooth. The metaconid is absent. The distal end of the tooth is broken off. A small hypoconid must have existed there.

The trigonid of the p4 is fully molarized, but the talonid is very poorly developed. The protoconid, paraconid and metaconid are all trenchant and tall. The protoconid is the largest cusp of the tooth. The paraconid is mesiolingually positioned, well separated from the protoconid. The metaconid is directly lingual relative to the protoconid. It is much larger than the paraconid, but considerably smaller than the protoconid. The paracristid, which connects the protoconid and paraconid, is very sharp. A deep notch is present at the middle of the paracristid, thereby forming a carnassial-like shearing blade. The lingual and buccal protocristids between the protoconid and metaconid are short, but sharp. The paraconid and the metaconid are widely separated from each other. The distal surface of the paraconid and the mesial surface of the metaconid are smooth. No cristid is present between these two cusps. The trigonid completely opens to the lingual side. The talonid is very short, bearing only one small cusp. This cusp is identified as the rudimentary hypoconid. The hypoconid is lingually positioned, distal to the metaconid rather than the protoconid. The distobuccal side of the tooth is thereby oblique. From the tip of the hypoconid, there are two weak cristids running to the mesiobuccal and mesiolingual borders of the trigonid, respectively. An even weaker cristid runs directly mesial to the posterior wall of the trigonid. This cristid represents the rudimentary cristid obliqua. A weak cingulid is developed on the mesiobuccal side of the tooth.

Two incomplete lower molars were identified as $\mathrm{m} 2 \mathrm{~s}$. The trigonid of this tooth is slightly mesiodistally compressed. The protoconid is in a triangular-pyramid shape.
Its distal and lingual sides are very flat and steep. Its mesiobuccal side is slightly curved. The metaconid is slender and lower than the protoconid. It is located directly lingual to the protoconid. The paraconid is much lower than the metaconid. Similar to paraconid of the lower molar of Tupaia, the paraconid of $P$. engesseri is also cristid-like. It is mesiodistally thin and buccolingually wide. Only its lingual side is conical. The paracristid developed on the mesial side of the protoconid is sharp and long. It starts at the tip of the protoconid, and runs mesiolingually to join the cristid-like paraconid. From the mesiobuccal view, the paracristid and the paraconid form a long and curved shearing blade. The buccal and lingual protocristids connect the protoconid and the metaconid, and form a V-shaped cutting edge. The paraconid is lingually positioned, almost directly mesial to the metaconid. A deep valley separates the two cusps. No cristid is developed between them. The talonid is as wide and long as the trigonid, but much lower. The hypoconid is in a triangular-pyramid shape with a broad base. Its mesiobuccal and distal side is flat, whereas the lingual side is slightly concave. Two cristids bearing on the hypoconid, the cristid obliqua and the hypocristid, are sharp and long. The former extends mesially to connect the distal wall of the trigonid near the base between the metaconid and protoconid, leaving a very deep hypoflexid on the buccal side of the tooth. The latter extends distolingually to join the hypoconulid. The entoconid is much smaller than the hypoconid. The preentoconid cristid is low but complete. It closes the talonid basin from the lingual side. The hypoconulid is relatively big, although its absolute size is smaller than the entoconid. It is located distal to the entoconid, and projects distally. The distal margin of the entoconid and the mesial margin of the hypoconulid are smooth. A broad valley separates the two cusps. A strong cingulid is developed on the mesiobuccal side of the trigonid. It extends from the base of the paraconid to the buccal side of the protoconid. No cingulid is present on the buccal, lingual and distal sides of the tooth.

An incomplete $\mathrm{m} 3$ shows that the tooth is very similar to $\mathrm{m} 2$, but smaller and having a narrower talonid.

Comparison The small tree shrew specimens collected from Yuanmou Lufengpithecus locality are slightly smaller than those of $P$. yunnanica (Table 1) and all the extant species of Dendrogale. The features that distinguish $P$. yunnanica from Dendrogale are mostly shared by $P$. yunnanica and the Yuanmou tree shrew specimens. These shared characters by the two species assure us that they should be assigned in the same genus.

Differences do exist when they are compared in detail. An incomplete specimen (V 8282.9) of $P$. yunnanica indicates that the $\mathrm{P} 4$ paracone of this species is much taller than that of $P$. engesseri sp. nov. (Fig. 2). The parastyle of 
$P$. yunnanica is much smaller and less anteriorly protruding. The indentation on the mesial and buccal margins of P. yunnanica is shallower. The $\mathrm{P} 4$ protocone of $P$. yunnanica is not preserved, but from the broken edge, we can deduce that it should be proportionally as large as that of $P$. engesseri.

The M1 or M2 of P. yunnanica has sharper and higher cusps, more protruding parastyle and mesostyle, and bigger paraconule and metaconule. The valley between the paracone and metacone is wider than that of $P$. engesseri. The M1 of $P$. yunnanica lacks the short cingulum on the distal side of the protocone. The M3 of $P$. yunnanica is very similar to that of $P$. engesseri, but it has a more elongated mesiobuccal corner.

The $\mathrm{p} 4$ of $P$. yunnanica is proportionally much bigger than that of $P$. engesseri (Fig. 2). The mesiobuccal side of the protoconid of the $P$. yunnanica is more bulging. The paraconid, however, is relatively weaker and lower.

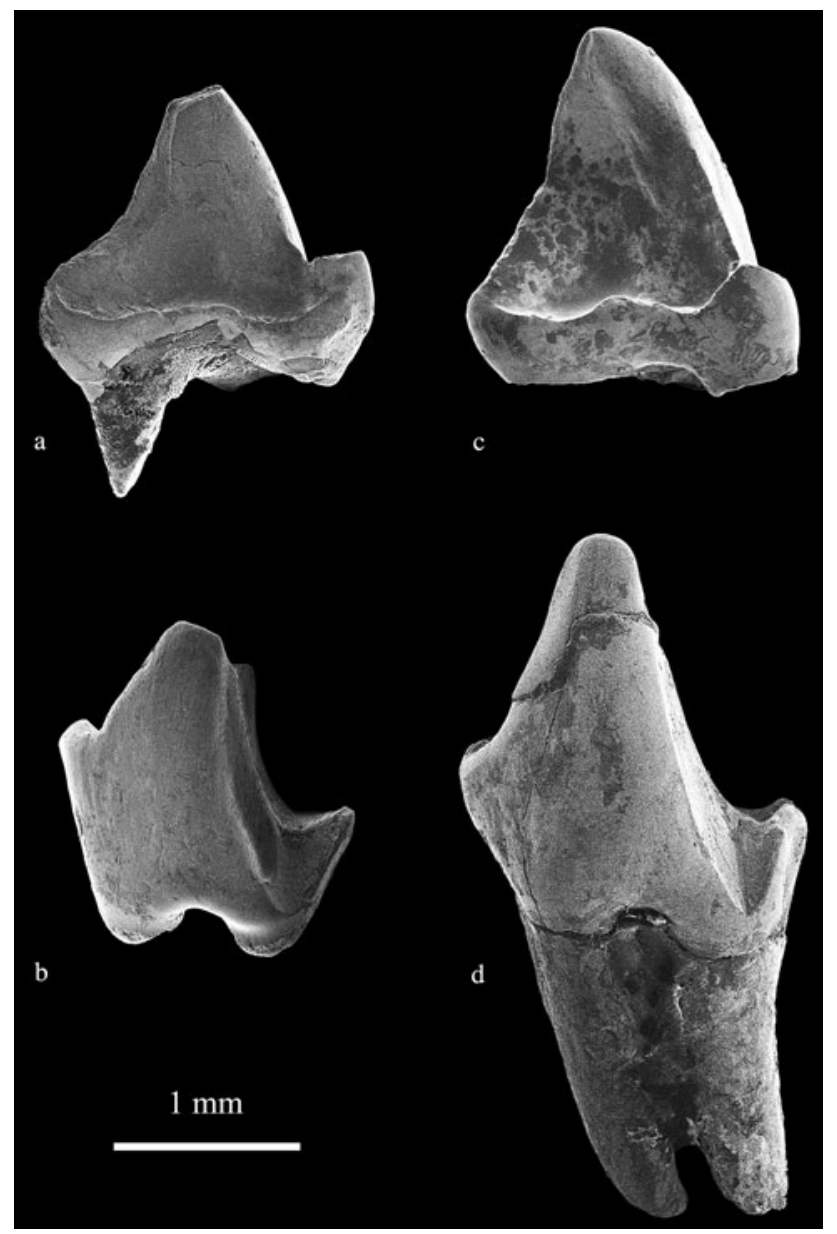

Fig. 2 Comparison of the buccal view of the P4 and p4. a Prodendrogale engesseri sp. nov., left P4, IVPP V 18216.2; b Prodendrogale engesseri sp. nov., left p4, IVPP V 18217.3; c P. yunnanica, left P4, IVPP V 8282.9; d P. yunnanica, left p4, IVPP V 8282.16
The lower molar of $P$. engesseri is also very similar to that of $P$. yunnanica, but the former is smaller and has lower crown. The hypoconulid of $P$. engesseri is more distally projecting than that of $P$. yunnanica. The valley separating the hypoconulid and the entoconid in $P$. engesseri is relatively wider.

Genus Tupaia Raffles 1822

Tupaia storchi sp. nov.

Figure 3a-c

Holotype Left m3 IVPP V 18218 (2.51 mm long, $1.60 \mathrm{~mm}$ trigonid wide, and $1.25 \mathrm{~mm}$ talonid wide, Fig. 3a)

Type locality Yuanmou Lufengpithecus locality 9906, Late Miocene, Leilao Village, Yuanmou County, Yunnan Province, China.

Etymology Dedicated to Dr. Gerhard Storch, in recognition of his outstanding work on fossil mammals and his important contributions to Chinese mammal palaeontology.

Referred specimens Right P4 IVPP V 18219.1 (2.35 mm long, and $2.50 \mathrm{~mm}$ wide); right p4 IVPP V 18219.2 (2.20 mm long, and $1.35 \mathrm{~mm}$ wide).

Diagnosis Medial-sized tree shrew. Larger than Palaeotupaia sivalicus, Tupaia minor and $T$. javanica, but smaller than all the other extant species and T. miocenica. P4 protocone conical with very round lingual side, parastyle large and mesially protruding, buccal cingulum incomplete. $\mathrm{m} 3$ talonid relatively broad and shallow, and mesiobuccal cingulid strong and long.

Description The P4 is triangular in the occlusal view, with straight buccal and distal borders and slightly concave mesial border. It possesses two main cusps, the paracone and protocone. The paracone is tall and sharp, much more prominent than the protocone. Its buccal side is rounded, pillar-like and near perpendicular to the buccal tooth border, but its lingual side is strongly slopping and much longer and broader than the buccal side. The preparacrista of the paracone is quite strong. It starts at the tip of the paracone and joins the parastyle. The postparacrista is much sharper, stronger and longer than the preparacrista. The mesial half of the postparacrista is slightly lingually orientated. The distal half the postparacrista switches to the distobuccal corner of the tooth, and forms a high and sharp shearing blade. From the lateral view, the paracone is distally inclined, with its preparacrista forming the convex border and its postparacrista forming the concave border. The protocone is mesiolingually positioned relative to the paracone. The lingual side of the protocone is round and bulging, whereas the buccal side is slightly concave. The preprotocrista is very strong, but blunt. It runs mesiobuccally for a very short distance to join the mesial cingulum. The postprotocrista is absent, but the distal surface of the protocone bears a short and blunt "Nannopithex"-fold. 


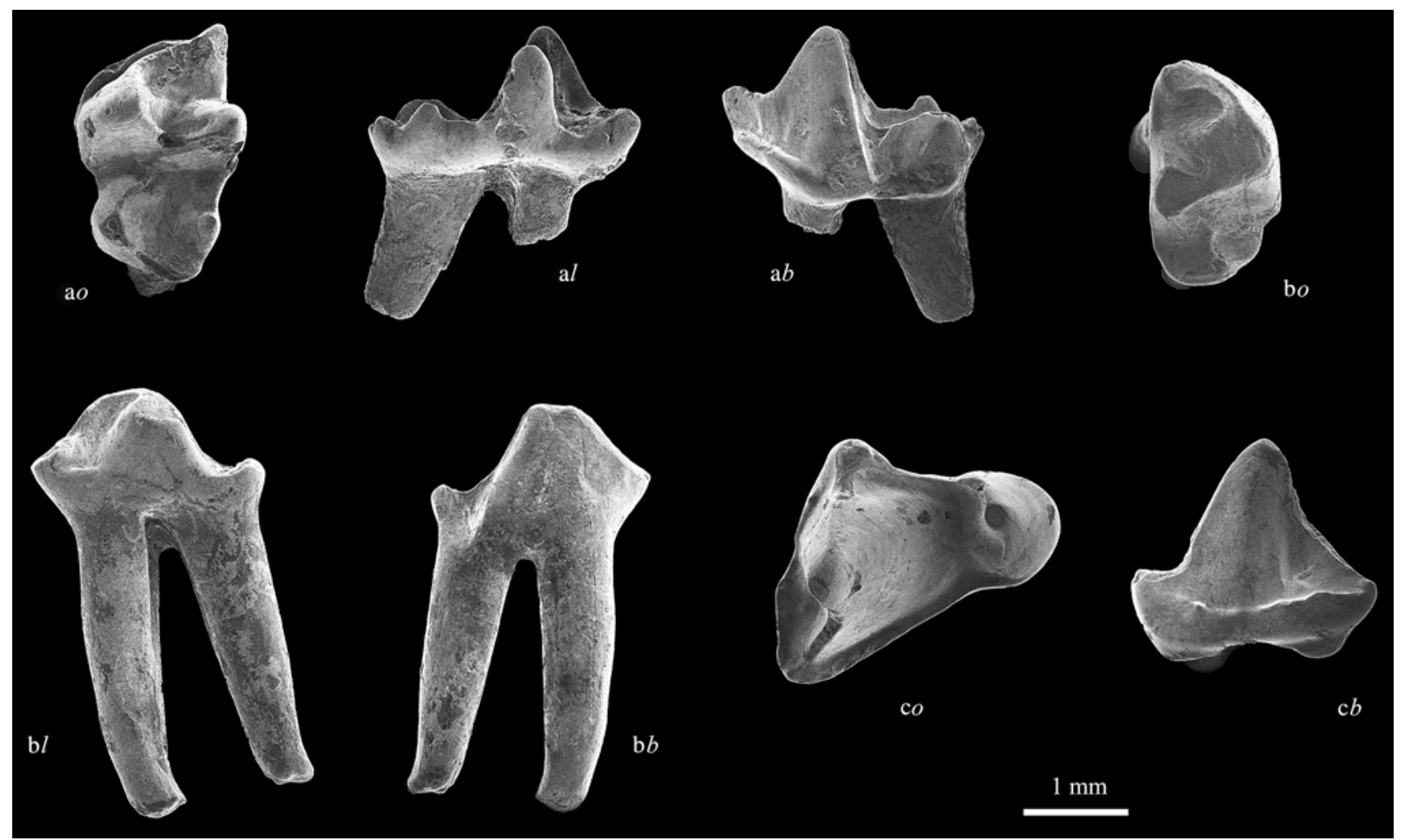

Fig. 3 Isolated teeth of Tupaia storchi sp. nov.: a left m3, IVPP V 18218, type; b right p4 IVPP V 18219. 2; c right P4 IVPP V 18219.1. $o$ occlusal view, $l$ lingual view, $b$ buccal view

This fold runs down from the tip of the protocone, and terminates before joining the distal cingulum. The parastyle is large and protruding mesially. The metastyle is very small, present as a small nodule. The mesial and distal cingula are very strong. The mesial half of the buccal cingulum is relatively weak, but the distal half is very strong. A narrow stylar shelf is formed between the distal half of the buccal cingulum and the distal crista of the paracone. The mesial and buccal cingula mesiobuccally connect the parastyle, whereas the distal and buccal cingula join each other distobuccally at the metastyle.

The p4 available for description is deeply worn. The wearing facet indicates that the protoconid was tall. The paraconid and metaconid are about the same size. Both are much lower than the protoconid. Mesiodistally, the paraconid is far mesially positioned, whereas the metaconid is very close to the protoconid and only slightly distal relative to the latter cusp. Buccolingually, both paraconid and metaconid are very close to the lingual border of the tooth. The trigonid valley between the paraconid and the metaconid is completely open to the lingual side. A very weak lingual cingulid is present between the paraconid and metaconid, but it does not join the two cusps. The paracristid, which connects the protoconid and the paraconid, must have been very strong. It probably forms a notched cutting edge before worn down. The protocristid should also be very strong, but obviously shorter than the paracristid. The distobuccal surface of the protoconid is round and smooth. No cristid is generated there. The distal surface of the paraconid, and the mesial and distal surfaces of the metaconid are round and smooth too. The talonid of the tooth is very short and not fully developed. The hypoconid is a small heel-like cusp, centrally located. A short and low cristid, probably homologous to the cristid obliqua, is generated from the hypoconid and extends to the middle of the distal wall of the trigonid. The hypoconulid and entoconid are absent, and the talonid basin is not developed yet. No cingulid is present on the lingual or buccal side.

The $\mathrm{m} 3$ trigonid is tall with sharp cusps. The protoconid is roughly in a shape of triangular pyramid, with a slightly curved mesiobuccal side, very flat distal side and slightly concave lingual side. A rib-like enamel ridge is developed on the lingual side of the protoconid. The metaconid is also in a shape of triangular pyramid, but more rounded and slender than the protoconid. The paraconid is much lower than the protoconid and the metaconid. From lingual view, it is a procumbent cusp, but from the occlusal and buccal view, it is more like a cristid than a cusp. The paracristid is very sharp. It runs from the tip of the protoconid, joins the cristid-like paraconid, and forms L-shaped shearing blade. 
The lingual and buccal protocristid is low and blunt. A V-shaped notch is formed between the protoconid and metaconid. The paraconid and metaconid are widely separated from each other. No cristid or ridge joins the two cusps. The trigonid basin is therefore fully opened to the lingual side. The talonid is much narrower and lower than the trigonid. The hypoconid is low and blunt. The cristid obliqua extends mesially from this cusp, and connects the distal wall of the trigonid at a point between the protoconid and the metaconid. Forming about a right angle with the cristid obliqua, the hypocristid extends distolingually to a point distal to the entoconid. The lingual end of the hypocristid is rounded and slightly elevated, it thereby can be called a hypoconulid. A shallow groove separates the hypoconulid and the entoconid. The entoconid is slightly higher, but much thinner than the hypoconid. Mesially the entoconid is confluent with the preentoconid cristid, and connects the metaconid along the lingual margin of the talonid. The talonid notch is fully closed by the preentoconid cristid. A very strong cingulid is developed along the tooth base on the mesiobuccal side of the trigonid. It origins from a point right below the paraconid, and extends distally to the buccal side of the protoconid. No cingulid is developed on the buccal, distobuccal and lingual sides of the tooth.

Comparison The specimens are assigned to the genus Tupaia based on a combination of straight buccal margin of P4, moderately developed P4 protocone, relatively broad p4 talonid, and relatively broad talonid basin of $\mathrm{m} 3$. In extant Dendrogale and the fossil Prodendrogale, the postparacrista of the $\mathrm{P} 4$ is long and buccally expanded. A prominent indentation is therefore generated on the buccal side of the tooth. In Anathana, Urogale and Tupaia, the buccal side of the $\mathrm{P} 4$ is straight, without clear indentation. The straight buccal side of $\mathrm{P} 4$ of $T$. storchi sp. nov. resembles the situation in three genera. In Dendrogale, Prodendrogale and Tupaia tana Raffles, 1821, the protocone of P4 is relatively narrow and short, whereas that of the $\mathrm{P} 4$ of $T$. storchi is moderately developed, proportionally broader and longer, but not as long and broad as those of Anathana and Urogale. In Prodendrogale, extant Dendrogale, Anathana and Urogale, the talonid of $\mathrm{p} 4$ is more lingually positioned and buccolingually much narrower than that of Tupaia. The distal border of the $\mathrm{p} 4$ of Tupaia is round, whereas in all the other genera, this border is pointed. The $\mathrm{p} 4$ of $T$. storchi has a relatively better developed talonid than all the other genera of Tupainae with the exception of Tupaia.

Within the genus Tupaia, T. storchi is larger than $T$. minor and $T$. javanica, but smaller than all the other extant species. The mesiobuccal cingulid of the lower molar of T. storchi is very strong. T. tana was assigned to the genus Tana or Lyonogale (Lyon 1913; Conisbee 1953), but it is generally accepted as a species of Tupaia now (McKenna and Bell 1997; Helgen 2005). Dentally, however, $T$. tana has a mesiodistally shorter protocone on the P4 and weaker hypoconulid on p4 than all the other species of Tupaia. These two features also distinguish $T$. tana from T. storchi. Mein and Ginsburg (1997) report an isolated left M2 of tree shrew discovered from the early Miocene Li Mae Long locality of Thailand. The specimen was named as T. miocenica. This species is much larger than all the other species of Tupaia, including T. storchi.

Some researchers suggested that the Miocene tree shrew Palaeotupaia from the Siwaliks of India is virtually identical to Tupaia and should not be allocated in a separated genus (Luckett and Jacobs 1980). The type specimen (PUA I-3) represents an individual about the size of modern T. minor, therefore smaller than T. storchi.

\section{Discussion}

The family Tupaiidae includes two subfamilies, Tupaiinae and Ptilocercinae. The ptilocercine fossils were found only from the Yuanmou Lufengpithecus locality ( $\mathrm{Ni}$ and Qiu 2002), but the specimens are yet to be studied in detail. The remains of tupaiines were discovered in Pakistan, India, Thailand and China. Although sparse, two evolutionary lineages, the Tupaia-lineage and the Dendrogale-lineage, could be distinguished. Chopra et al. (1979; Chopra and Vasishat 1979) reported a skull fragment preserving the facial part and most of the upper dentition discovered in the Siwalik deposits of Haritalyangar in Himachal Pradesh, India. The specimen (PUA I-3) is the most complete tree shrew fossil ever found. PUA I-3 plus a maxillary fragment (PUA I-5) preserving left M1-3 and an isolated right m2 (PUA I-6) were named as $P$. sivalicus, implying an ancient form of Tupaia (Chopra et al. 1979; Chopra and Vasishat 1979). Luckett and Jacobs (1980), however, argued that no essential craniodental differences exist between Tupaia and Palaeotupaia. Two specimens referred to $P$. sivalicus, PUA I-5 and PUA I-6, from the same locality have about the same body size as that of the type specimen. But PUA I-5 probably represents a different species, because its M1 and M2 have a much more prominent hypocone (Qiu 1986). PUA I-6 is a lower molar. It is hard to tell to which form it could belong. The only fossil species from Thailand, Tupaia miocenica, is represented by only one M2 (Mein and Ginsburg 1997). The specimen has a quite elongated postmetacrista and mesially inclined protocone. We believe it is an M1 instead of M2. The Thailand tree shrew is distinct from all the other Tupaia species by its very large size, even larger than the largest extant tree shrew T. tana. Tupaia storchi sp. nov. reported here, added another member to the Tupaia-lineage. It is distinct from 
all the other species by possessing a very strong mesiobuccal cingulid on the lower molar. This character probably represents a primitive feature. In a short communication, Dutta (1975) briefly mentioned a possible Tupaia rib cage discovered from the Pliocene Tatrot beds of the upper Siwaliks of India. Although this report has been widely cited, it is hard to evaluate the referral of this rib cage to Tupaia without sufficient information of morphological details.

The fossil records of Dendrogale-lineage include the specimens referred to Prodendrogale and some unnamed specimens from the Pakistan Siwaliks. Jacobs (1980) reported an anterior skull fragment of tree shrew, an isolated lower molar and a talonid of a lower molar from three Yale-Geological Survey of Pakistan expedition localities in the Siwaliks of Pakistan. These specimens may represent a new genus and new species (Jacobs 1980; Sargis 2004). The skull fragment belongs to a species comparable in size to Tupaia glis (Diard, 1820), but morphologically is quite generalized. It shares many similarities with Tupaia, Dendrogale, Urogale, Anathana, and even Ptilocercus. However, Jacobs (1980) believed that the skull fragment probably is more similar to Dendrogale than to other species in having double-rooted canines. All the teeth of the skull fragment are not preserved. It is thereby impossible to compare it with other species based on dental characters. Qiu (1986) named P. yunnanica based on some isolated teeth discovered from the Lufeng Lufengpithecus locality. Prodendrogale is very close to the extant Dendrogale, and the two genera probably have sister-group relationships. P. engesseri sp. nov. from Yuanmou Lufengpithecus locality is remarkably similar to $P$. yunnanica. Smaller size, relatively lower crown and less trenchant cusps of $P$. engesseri suggest a more primitive condition than $P$. yunnanica. This conclusion is consistent with its older age estimated based on small mammalian fauna correlations (Ni and Qiu 2002). Tong (1988) described five tiny tooth fragments discovered from the middle Eocene Hetaoyuan Formation of Henan Province, China, and raised the name Eodendrogale parva, implying an Eocene form of Dendrogale. Unfortunately, the author cannot find these specimens now (personal communication). The illustration of the specimens published by Tong (1988) does not provide sufficient information for judging the systematic allocation of E. parva to the order Scandentia. The upper molar of $E$. parva probably has a distinct distal stylar cusp. The talonid basin is very narrow. The hypoconulid is very strong. The distal cingulid is strong, almost like a shelf, but the buccal cingulid is absent. The combination of these features is very unusual for tree shrews.

The distributions of extant Tupaia, Dendrogale and Ptilocercus only overlap on the Borneo Island (Helgen 2005; Lyon 1913). However, the discovery of diverse tree shrews from the same locality in Yunnan may suggest that different tree shrew lineages must have evolved and coexisted in a much larger area than their current distributions.

Acknowledgments We thank Professors Qi Guoqin, Pan Yuerong, Dr. Zhao Lingxia, Messrs. Zheng Liang, Gao Feng, Ji Xueping, Liu Jianhui, Jiang Chu, and Zhang Jiahua for their assistance in the field. Ms. Wang Guizhen sorted the concentrates. Mr. Zhang Wending took the SEM photos. We extend our gratitude to the anonymous reviewers for providing helpful comments and suggestions. This project has been financially supported by Chinese Academy of Sciences (CAS) Young Scholar Project, CAS 100-Talent Project, CAS Fossil Excavation and Preparation Fund, National Basic Research Program of China (2012CB821904) and the National Natural Science Foundation of China (NSFC 40672009, 40872032).

\section{References}

Bloch, J. I., Silcox, M. T., Boyer, D. M., \& Sargis, E. J. (2007). New Paleocene skeletons and the relationship of plesiadapiforms to crown-clade primates. PNAS, 104, 1159-1164.

Carlsson, A. (1922). Über die Tupaiidae und ihre Beziehungen zu den Insectivora und den Prosimiae. Acta Zoologica, 3, 227-270.

Chopra, S. R. K., Kaul, S., \& Vasishat, R. N. (1979). Miocene tree shrews from the Indian Śivaliks. Nature, 281, 213-214.

Chopra, S. R. K., \& Vasishat, R. N. (1979). Śivalik fossil tree shrew from Haritalyangar, India. Nature, 281, 214-215.

Conisbee, L. R. (1953). A list of the names proposed for genera and subgenera of recent mammals, from the publication of T. S. Palmer's Index Generum Mammalium, 1904 to the end of 1951. London: British Museum (Natural History).

Dutta, A. K. (1975). Micromammals from Siwaliks. Indian Minerals, 29, 76-77.

Helgen, K. M. (2005). Order Scandentia. In D. E. Wilson \& D. M. Reeder (Eds.), Mammal species of the world. A taxonomic and geographic reference. Third edition (pp. 104-109). Baltimore: The Johns Hopkins University Press.

Jacobs, L. L. (1980). Siwalik fossil tree shrews. In W. P. Luckett (Ed.), Comparative biology and evolutionary relationships of tree shrews (pp. 205-216). New York: Plenum Press.

Janecka, J. E., Miller, W., Pringle, T. H., Wiens, F., Zitzmann, A., Helgen, K. M., et al. (2007). Molecular and genomic data identify the closest living relative of primates. Science, 318, 792-794.

Le Gros Clark, W. E. (1924a). The myology of the tree-shrew (Tupaia minor). Proceedings of the Zoological Society of London, 94, 461-497.

Le Gros Clark, W. E. (1924b). On the brain of the tree-shrew (Tupaia minor). Proceedings of the Zoological Society of London, 94, 1053-1074.

Le Gros Clark, W. E. (1925). On the skull of Tupaia. Proceedings of the Zoological Society of London, 95, 559-567.

Le Gros Clark, W. E. (1926). On the anatomy of the pen-tailed treeshrew (Ptilocercus lowii). Proceedings of the Zoological Society of London, 96, 1179-1309.

Luckett, W. P., \& Jacobs, L. L. (1980). Proposed fossil tree shrew genus Palaeotupaia. Nature, 288, 104.

Lyon, M. W. (1913). Treeshrews: an account of the mammalian family Tupaiidae. Proceedings of the United States National Museum, 45, 1-188.

Martin, R. D. (1968). Towards a new definition of primates. Man, 3, 377-401. 
Matthew, W. D., \& Granger, W. (1924). New insectivores and ruminants from the Tertiary of Mongolia, with remarks on the correlation. American Museum Novitates, 105, 1-7.

McKenna, M. C. (1966). Paleontology and the origin of the primates. Folia Primatologica, 4, 1-12.

McKenna, M. C., \& Bell, S. K. (1997). Classification of mammals, above the species level. New York: Columbia University Press.

Mein, P., \& Ginsburg, L. (1997). Les mammifères du gisement miocène inférieur de Li Mae Long, Thaillande : systématique, biostratigraphie et paléoenvironnement. Geodiversitas, 19, 783-844.

Ni, X., Meng, J., Beard, K. C., Gebo, D. L., Wang, Y., \& Li, C. (2010). A new tarkadectine primate from the Eocene of Inner Mongolia, China: phylogenetic and biogeographic implications. Proceedings of the Royal Society B: Biological Sciences, 277, 247-256.

Ni, X., \& Qiu, Z. (2002). The micromammalian fauna from the Leilao, Yuanmou hominoid locality: Implications for biochronology and paleoecology. Journal of Human Evolution, 42, 535.

Olson, L. E., Sargis, E. J., \& Martin, R. D. (2004). Phylogenetic relationships among treeshrews (Scandentia): A review and critique of the morphological evidence. Journal of Mammalian Evolution, 11, 49-71.

Olson, L. E., Sargis, E. J., \& Martin, R. A. (2005). Intraordinal phylogenetics of treeshrews (Mammalia: Scandentia) based on evidence from the mitochondrial $12 \mathrm{~S}$ rRNA gene. Molecular Phylogenetics and Evolution, 35, 656-673.

Qi, G., \& Dong, W. (2006). Lufengpithecus hudienensis Site. Beijing: Science Press.

Qiu, Z. (1986). Fossil tupaiid from the hominoid locality of Lufeng, Yunnan. Vertebrata PalAsiatica, 24, 308-319.

Sargis, E. J. (2004). New views on tree shrews: the role of tupaiids in primate supraordinal relationships. Evolutionary Anthropology, $13,56-66$.

Schmitz, J., Ohme, M., \& Zischler, H. (2000). The complete mitochondrial genome of Tupaia belangeri and the phylogenetic affiliation of Scandentia to other eutherian orders. Molecular Biology and Evolution, 17, 1334-1343.

Simmons, N. B. (1993). The importance of methods: Archontan phylogeny and cladistic analysis of morphological data. In R. D. E. MacPhee (Ed.), Primates and their relatives in phylogenetic perspective (pp. 1-61). New York: Plenum Press.

Simpson, G. G. (1931). A new insectivore from the Oligocene, Ulan Gochu horizon, of Mongolia. American Museum Novitates, 505, $1-22$.

Simpson, G. G. (1945). The principles of classification and a classification of mammals. Bulletin of the American Museum of Natural History, 85, 1-350.

Springer, M. S., Burk-Herrick, A., Meredith, R., Eizirik, E., Teeling, E., O'Brien, S. J., et al. (2007). The adequacy of morphology for reconstructing the early history of placental mammals. Systematic Biology, 56, 673-684.

Steele, D. G. (1973). Dental variability in the tree shrews (Tupaiidae). In M. R. Zingeser (Ed.), Craniofacial Biology of Primates (pp. 154-179). Basel: S. Karger.

Swindler, D. R. (1976). Dentition of living primates. London: Academic Press.

Szalay, F. S. (1968). The beginnings of primates. Evolution, 22, 19-36.

Szalay, F. S. (1977). Phylogenetic relationships and a classification of the eutherian Mammalia. In M. K. Hecht, P. C. Goody, \& B. M. Hecht (Eds.), Major patterns in vertebrate evolution (pp. 315-374). New York: Plenum Press.

Szalay, F. S., \& Delson, E. (1979). Evolutionary history of the primates. London: Academic Press.

Tong, Y. (1988). Fossil tree shrews from the Eocene Hetaoyuan Formation of Xichuan, Henan. Vertebrata PalAsiatica, 26, 214-220.

Van Valen, L. (1965). Treeshrews, primates, and fossils. Evolution, 19, 137-151. 